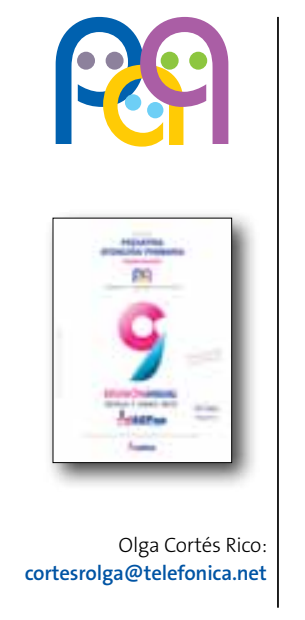

\title{
Tratamiento del asma
}

O. Cortés Rico

Pediatra. CS Canillejas, Madrid. España. Grupo de Vías Respiratorias de la AEPap

\section{INTRODUCCIÓN}

El asma es una enfermedad que se puede controlar, de forma mantenida, con medidas ambientales y medicamentos.

Los beneficios del tratamiento con fármacos para el control del asma superan con creces los riesgos de efectos adversos.

El pediatra de Atención Primaria (AP) puede dirigir el tratamiento de la mayor parte de los niños y adolescentes con asma.

\section{¿CUÁNDO ESTÁ INDICADO INICIAR TRATAMIENTO CONTROLADOR?}

La inflamación de la vía aérea se halla presente en todos los asmáticos, sea cual sea su edad y grave$\mathrm{dad}^{1}$. El remodelamiento de la vía aérea también se inicia en las fases tempranas de la enfermedad y puede condicionar en determinados pacientes una obstrucción irreversible al flujo aéreo².

De ahí la importancia del tratamiento de fondo, de mantenimiento o controlador, el cual se basa en cuatro pilares básicos: a) educación del paciente y su familia; b) control medioambiental, con medidas de evitación de desencadenantes; c) tratamiento farmacológico, y d) monitorización y seguimiento.
El objetivo del tratamiento del asma (Tabla 1) es mantener el control de la enfermedad ${ }^{1,3,4}$ y se identifican dos componentes fundamentales del control $^{4,5}$ : alcanzar el control actual y disminuir el riesgo futuro. Alcanzar el control actual en cuanto a síntomas, limitación de actividades, uso de la medicación de rescate y función pulmonar. El riesgo futuro es el de presentar crisis, hospitalizaciones, deterioro irreversible de la función pulmonar o efectos adversos de la medicación.

Tabla 1. Objetivos del tratamiento del asma

- Control actual:

- Prevenir los síntomas diurnos, nocturnos y tras ejercicio físico

- Uso de agonista de acción corta no más de dos días a la semana

- Mantener una función pulmonar normal o casi normal

- Sin restricciones en la vida cotidiana y para realizar ejercicio físico

- Cumplir las expectativas de los pacientes y sus familias

- Riesgo futuro:

- Prevenir las exacerbaciones y la mortalidad

- Minimizar la pérdida progresiva de la función pulmonar

- Evitar los efectos adversos del tratamiento

GEMA 2009. 


\section{¿CÓMO INICIAR EL TRATAMIENTO CONTROLADOR?}

La clasificación del asma por gravedad se debe realizar cuando el paciente está sin tratamiento y es útil para decidir el tratamiento de mantenimiento inicial (Tablas 2 y 3).

Posteriormente será la evolución clínica y la consecución de los objetivos de control los que dicten las modificaciones del tratamiento.
En los niños con asma episódica ocasional, el tratamiento se realizará con beta adrenérgicos de acción corta a demanda, sin tratamiento de mantenimiento, escalón 1. En los niños con asma episódica frecuente se aconseja iniciar el tratamiento en el escalón 2. Los niños con asma persistente moderada en el escalón 3. En los niños con asma grave es preferible iniciar el tratamiento en el escalón 5 y en cuanto se logre el control bajar de escalón, buscando siempre la dosis mínima efectiva (Tablas 4 y 5 ).

\begin{tabular}{|c|c|c|c|c|}
\hline \multirow{2}{*}{\multicolumn{2}{|c|}{ Gravedad del asma }} & \multicolumn{2}{|c|}{ Control de base de la enfermedad } & \multirow{2}{*}{ Alivio síntomas } \\
\hline & & Elección & No precisa & \\
\hline \multicolumn{2}{|l|}{ Episódica ocasional } & No precisa & No precisa & \multirow{6}{*}{$\begin{array}{l}\text { BAAC } \\
\text { a demanda }\end{array}$} \\
\hline \multirow[t]{2}{*}{ Episódica frecuente } & IPA - & Habitualmente no precisa & $\begin{array}{l}\text { Valorar respuesta: } \\
\text { - ARLT } \\
\text { - GCI dosis bajas }\end{array}$ & \\
\hline & IPA + & GCI dosis bajas & ARLT & \\
\hline \multirow{2}{*}{\multicolumn{2}{|c|}{$\begin{array}{l}\text { Persistente moderada } \\
\text { (antes de dar este paso es preciso } \\
\text { replantearse el diagnóstico y la adecuada } \\
\text { administración del tratamiento) }\end{array}$}} & GCl dosis medias & $\begin{array}{l}\text { GCl dosis medias + } \\
\text { ARLT }\end{array}$ & \\
\hline & & \multicolumn{2}{|c|}{$\begin{array}{l}\text { Valorar respuesta a los tres meses. Retirar si no hay } \\
\text { respuesta y si no existen factores de riesgo }\end{array}$} & \\
\hline \multicolumn{2}{|l|}{ Persistente grave } & \multicolumn{2}{|c|}{$\begin{array}{l}\mathrm{GCI} \text { dosis altas } \\
\text { Se pueden considerar una o varias: } \\
\text { ARLT, BAAL o GC oral }\end{array}$} & \\
\hline
\end{tabular}

ARLT: antagonistas de los receptores de leucotrienos; BAAC: agonistas beta de acción corta; BAAL: agonistas beta de acción larga; GC: glucocorticoides; GCl: corticoides inhalados.

Consenso y GEMA 2009.

\section{Tabla 3. Tratamiento inicial en mayores de 3 años (Consenso y GEMA 2009)}

\begin{tabular}{|c|c|c|c|c|}
\hline \multirow{2}{*}{$\begin{array}{c}\text { Gravedad inicial } \\
\text { del asma }\end{array}$} & \multicolumn{2}{|c|}{ Medicación control } & \multirow{2}{*}{ Inmunoterapia } & \multirow{2}{*}{ Mediación de rescate } \\
\hline & Elección & Alternativa & & \\
\hline Episódica ocasional & No precisa & No precisa & & \multirow{4}{*}{ BAAC a demanda } \\
\hline Episódica frecuente & GCI dosis bajas & ARLT & + & \\
\hline Persistente moderada & $\mathrm{GCl}$ dosis medias & $\begin{array}{l}\mathrm{GCl} \text { dosis bajas + BAAL } \\
\text { o } \\
\mathrm{GCl} \text { dosis bajas + ARLT }\end{array}$ & + & \\
\hline Persistente grave & \multicolumn{2}{|c|}{$\begin{array}{l}\text { GCl dosis media/alta + BAAL. Considerar añadir } \\
\text { uno o varios: GCO, ARLT, metilxantinas, Ac. } \\
\text { monoclonales anti-lge }\end{array}$} & & \\
\hline
\end{tabular}

Ac.: anticuerpos; ARLT: antagonistas de los receptores de leucotrienos; BAAC: agonistas beta de acción corta; BAAL: agonistas beta de acción larga; GCl: corticoides inhalados. 


\begin{tabular}{|c|c|c|c|c|}
\hline \multirow{7}{*}{$\begin{array}{l}+ \\
\text { C } \\
O \\
N \\
T \\
R \\
O \\
\text { R } \\
-\end{array}$} & \multirow{7}{*}{$\begin{array}{c}\text { Evaluación } \\
\text { del cumplimiento } \\
\text { y la técnica } \\
\text { inhalatoria } \\
\text { Control ambiental }\end{array}$} & $\begin{array}{l}\text { Tratamiento } \\
\text { escalonado }\end{array}$ & Medicación de control & $\begin{array}{l}\text { Medicación } \\
\text { de rescate }\end{array}$ \\
\hline & & 1 & Sin medicación & \multirow{6}{*}{ BAAC a demanda } \\
\hline & & 2 & $\begin{array}{c}\text { GCI dosis bajas } \\
\text { o ARLT }\end{array}$ & \\
\hline & & 3 & $\begin{array}{c}\text { GCl dosis medias } \\
\text { o GCl dosis bajas + ARLT }\end{array}$ & \\
\hline & & 4 & GCI dosis medias + ARLT & \\
\hline & & 5 & $\begin{array}{c}\text { GCI dosis altas + ARLT } \\
\text { Si no control añadir BAAL }\end{array}$ & \\
\hline & & 6 & GCO & \\
\hline
\end{tabular}

En negrita el tratamiento de elección. GEMA 2009.

¿CÓMO HACER CAMBIOS EN LA MEDICACIÓN DE CONTROL? ¿ES ÚTIL EL "GRADO DE CONTROL DEL ASMA" PARA MODIFICAR EL TRATAMIENTO DE FONDO?

Una vez iniciado el tratamiento de mantenimiento, las modificaciones se realizarán también de forma escalonada (Tabla 4 para menores de tres años, y Tabla 5 para los mayores de esta edad), subiendo o bajando un escalón en función del grado de control obtenido ${ }^{5}$ :

- Si el asma no está controlada con el régimen de tratamiento actual, debería subirse un escalón hasta conseguir el control, comprobando previamente si toma la medicación, si realiza corectamente la técnica de inhalación y si evita los posibles desencadenantes.

- Si se encuentra parcialmente controlada, habría que valorar avanzar en los pasos de tratamiento, considerando otras opciones disponibles y el grado de satisfacción del paciente con el control actual.

- Cuando se mantiene un buen control durante al menos tres meses, se puede bajar de escalón. La meta es lograr disminuir el tratamiento hasta alcanzar el mínimo con el que el paciente se mantenga controlado.

\section{Tabla 5. Tratamiento escalonado según control en mayores de tres años}

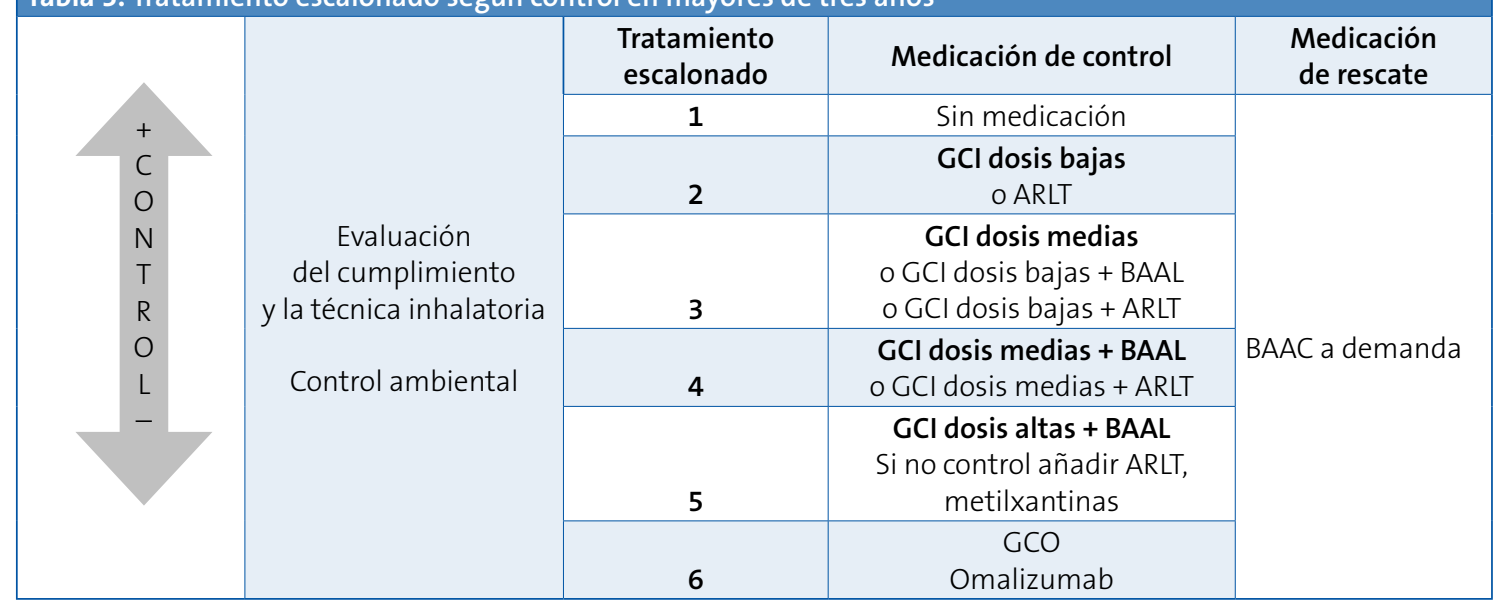

En negrita el tratamiento de elección. 
Una vez controlada el asma, es imprescindible una monitorización periódica del tratamiento para establecer el escalón más bajo necesario que nos permita mantener el control, minimizando el coste y los efectos adversos y maximizando la seguri$\mathrm{dad}^{1-5}$.

\section{¿CUÁL ES EL FÁRMACO DE ELECCIÓN PARA INICIAR TRATAMIENTO CONTROLADOR?}

Los glucocorticoides inhalados (GCl) son el tratamiento preventivo más efectivo del asma de distintos grados de gravedad $d^{3,4}$.

Son los fármacos recomendados de primera elección por todas las guías para conseguir globalmente los objetivos del tratamiento en todas las edades (Recomendación A), y se debería considerar su uso de forma temprana, incluso con función pulmonar normal.

Actúan inhibiendo la cascada inflamatoria: mejoran el control de los síntomas, mejoran la función pulmonar a largo plazo y previenen las reagudizaciones de asma con un perfil aceptable de seguridad $^{6}$. Además, disminuyen la pérdida de función pulmonar relacionada con las exacerbaciones graves de asma ${ }^{7}$.

Los corticoides inhalados en dosis bajas son superiores a la teofilina oral, el nedocromil, el cromoglicato sódico y los beta-2 de larga duración, para conseguir mejorar la función pulmonar, menor uso de beta-2 de alivio, mejorar la hiperreactividad bronquial y menor tasa de recaídas que precisen de corticoides orales ${ }^{1}$.

En cuanto a cuándo introducir el tratamiento con corticoides inhalados, en los últimos años hay acuerdo generalizado en comenzar el tratamiento controlador en el asma leve persistente (según cla- sificación de la Iniciativa Global para el Asma [GINA]) o episódica frecuente (según clasificación de la Guía Española de Manejo del Asma [GEMA] o del Consenso para el tratamiento del asma en Pediatría $)^{5,8}$. Y según el nuevo enfoque del control del asma, el tratamiento controlador se iniciará cuando la frecuencia y gravedad de los síntomas indiquen que el asma está parcialmente controlada o mal controlada ${ }^{1,7}$.

Se recomienda iniciar el tratamiento con $\mathrm{GCl}$ en dosis adecuada a la gravedad del asma estimada en ese momento, habitualmente dosis bajas o medias (Tabla 6), revisando la dosis cada 1-3 meses, para valorar el grado de control. Se puede probar como alternativa, fundamentalmente en los menores de cinco años, el tratamiento con montelukast, pasando a $\mathrm{GCl}$ si no se obtiene la respuesta adecuada.

\section{¿ANTILEUCOTRIENOS O CORTICOIDES INHALADOS?}

En niños de 2 a 14 años con asma persistente, el montelukast es seguro a corto plazo y produce una modesta mejoría frente a placebo ${ }^{3,4,9}$, también parece disminuir el número de crisis en niños con asma intermitente inducida por virus ${ }^{5,8,9}$.

Comparados con los $\mathrm{GCl}$, múltiples estudios han demostrado que los inhibidores de los leucotrienos son menos eficaces que los $\mathrm{GCl}$ en el control de los síntomas, las exacerbaciones del asma y el mantenimiento de la función pulmonar.

La GEMA ${ }^{5}$ los propone de inicio en menores de tres años con Índice Predictivo de Asma (IPA) negativo.

Se pueden plantear como tratamiento de inicio en aquellos niños pequeños con asma inducida por virus y exacerbaciones frecuentes. También se po-

Tabla 6. Dosis equipotentes en niños ( $\mu \mathrm{g} / \mathrm{di} i$ )

\begin{tabular}{|l|l|l|l|}
\hline & \multicolumn{1}{|c|}{ Dosis bajas } & \multicolumn{1}{c|}{ Dosis medias } & \multicolumn{1}{c|}{ Dosis altas } \\
\hline Beclometasona & $\leq 200$ & $200-400$ & $>400$ \\
\hline Budesonida & $\leq 200$ & $200-400$ & $>400$ \\
\hline Fluticasona & $\leq 100$ & $100-250$ & $>250$ \\
\hline
\end{tabular}


dría considerar su uso en niños menores de cuatro años que estén insuficientemente controlados con $\mathrm{GCl}$, puesto que los beta-agonistas de acción larga (BAL) no tienen indicación aprobada en estos niños. No hay estudios que comparen las dos alternativas como terapia añadida en niños.

Los antileucotrienos serían también una alternativa en pacientes que no pueden o no desean recibir glucocorticoides inhalados, que tienen efectos adversos con los mismos, o que tienen dificultades con la técnica de inhalación.

\section{¿SON SEGUROS LOS $\beta 2$ AGONISTAS DE ACCIÓN LARGA?}

En el momento actual, revisando las últimas publicaciones, incluida una revisión Cochrane, parece lo más sensato mantener una alerta sobre el uso de los BAL como medicación de primera línea en pacientes asmáticos. Su uso, por supuesto siempre en combinación con $\mathrm{GCl}$, debe reservarse para aquellos pacientes en los que no podamos alcanzar un buen control pese a aumentar las dosis de GCI hasta su límite terapéutico.

No existen estudios en menores de seis años de edad, por lo que no puede recomendarse el uso rutinario de las combinaciones por debajo de esta edad. Y hay que tener en cuenta que, en nuestro medio, según ficha técnica, el formoterol no está recomendado en niños menores de seis años y salmeterol no está recomendado en menores de cuatro.

\section{¿CUÁNDO HAY OQUE AÑADIR UN AGONISTA $\beta_{2}$ ADRENÉRGICO DE ACCIÓN LARGA AL GLUCOCORTICOIDE INHALADO?}

En la mayoría de los pacientes el efecto óptimo de los $\mathrm{GCl}$ se consigue con dosis bajas y medias; a partir de estas dosis, la curva dosis-respuesta es casi plana y sin embargo se incrementan de forma importante los efectos secundarios, por lo que una de las posibles opciones terapéuticas es añadir un BAL: salmetererol o formoterol. El beneficio de añadir un BAL es superior a aumentar la dosis de corticoide $^{1-3}$

La dosis de $\mathrm{GCl}$ a partir de la cual se recomienda introducir la terapia añadida, antes de aumentar la dosis de $\mathrm{GCl}$, no está del todo clara, pero en general las distintas guías recomiendan introducir un BAL cuando el control no es adecuado en dosis de 200$400 \mu \mathrm{g} /$ día de $\mathrm{GCl}^{1-6}$.

\section{¿HAY ALGÚN FÁRMACO OQUE MODIFIOQUE LA HISTORIA NATURAL DEL ASMA?}

La pérdida progresiva de la función pulmonar no se previene o no es del todo reversible con los corticoides inhalados 5 .

Los niños menores de tres años con sibilancias persistentes pueden controlarse con $\mathrm{GCl}$, pero no parece que el tratamiento modifique la evolución de la enfermedad.

La capacidad antiinflamatoria de los antagonistas de los leucotrienos es menor que la de los GCl.

Los BAL han demostrado mejoría de los síntomas y de la función pulmonar, pero no disminución de las exacerbaciones.

Las cromonas no han demostrado un efecto mejor que el placebo.

Sin embargo, puesto que la inflamación es un componente precoz y persistente en el asma, y parece tener un papel importante en el remodelamiento de la vía aérea, el tratamiento de mantenimiento debe ir dirigido a suprimir la inflamación, y se debe iniciar de forma precoz para evitar la disminución irreversible del flujo aéreo y el remodelamiento de la vía aérea.

\section{¿CÓMO SE VALORA LA GRAVEDAD DE LA CRISIS?}

La valoración de la gravedad de una crisis asmática se hace en función de signos clínicos, pruebas de función pulmonar (flujo espiratorio máximo o volumen espiratorio forzado) y medidas de oxigenación como la saturación de oxígeno ${ }^{1,2,10}$. 
También es importante tener en cuenta si el paciente ha precisado corticoides orales en crisis previas, el tiempo de evolución de la crisis (cuanto mayor sea el tiempo de evolución, peor será la respuesta al tratamiento), la medicación recibida, la duración del tratamiento previo con un beta-adrenérgico de acción corta y los antecedentes de riesgo para tener una crisis grave 2 .

Existen diversas escalas para valorar la gravedad de la crisis de asma. Una muy recomendable por su sencillez y aplicabilidad a todas las edades es el Pulmonary Score ${ }^{10}$ (Tabla 7).

\section{¿HAY QUE TRATAR CON CORTICOIDES ORALES TODAS LAS CRISIS DE ASMA?}

El objetivo del tratamiento de la crisis de asma es aliviar lo antes posible la obstrucción al flujo aéreo y la hipoxemia, y prevenir la aparición de futuras exacerbaciones.

Para ello se administrarán broncodilatadores de acción rápida (agonistas $B_{2}$ adrenérgicos de acción corta y anticolinérgicos), oxígeno suplementario y corticoides sistémicos para reducir la inflamación de las vías aéreas y prevenir recaidas ${ }^{1-6}$.

El uso precoz, durante la primera hora, de corticoides sistémicos durante las crisis es muy efectivo, reduciendo la tasa de ingresos a más de la mitad. Están indicados en todas las crisis moderadas y graves y también en el tratamiento de las crisis leves si con la dosis inicial de beta-adrenérgicos no se consigue una mejoría mantenida o en caso de que en las crisis previas hayan precisado el uso de un corticoide por vía sistémica. La vía oral es tan efectiva como la vía parenteral, por lo que se considera de elección siempre que sea bien tolerada.

La dosis recomendada es de 0,5-1 mg/ $\mathrm{kg} /$ día en crisis moderada, durante 3 a 5 días, y de $2 \mathrm{mg} / \mathrm{kg} /$ día en las crisis graves. No es necesario realizar descenso progresivo de dosis si se utilizan por debajo de diez días.

\section{¿CÓMO SE PUEDE MEJORAR LA CALIDAD DE LA ATENCIÓN AL PACIENTE CON ASMA?}

Las recomendaciones basadas en las guías de práctica clínica (GPC) no consiguen por sí solas una modificación de la asistencia ni una mejoría de la atención sanitaria.

Para ello es preciso conseguir la adherencia de los profesionales sanitarios implicados en la atención al niño con asma y definir unas normas de buena práctica clínica o criterios de buena atención.

Es necesario además su registro en la historia clínica, permitiendo de esta forma obtener indicadores útiles, tanto a los clínicos para mejorar la asistencia a su población infantil y adolescente con asma, como a los gestores sanitarios encargados de evaluar la calidad de la asistencia.

Por lo tanto, en la historia clínica de todo niño o adolescente con asma debe estar realizado y registrado:

\section{Tabla 7. Pulmonary score (PS) para valoración de la crisis de asma}

\begin{tabular}{|c|c|c|c|c|}
\hline \multirow{2}{*}{ Puntuación } & \multicolumn{2}{|c|}{ Frecuencia respiratoria } & \multirow{2}{*}{ Sibilancias } & \multirow{2}{*}{ Uso de músculo ECM } \\
\hline & $<6$ años & $\geq 6$ años & & \\
\hline 0 & $<30$ & $<20$ & No & No \\
\hline 1 & $31-45$ & $21-35$ & Final espiración & Incremento leve \\
\hline 2 & $46-60$ & $36-50$ & Toda la espiración & Aumentado \\
\hline 3 & $>60$ & $>50$ & Inspiración y espiración, sin estetoscopio & Actividad máxima \\
\hline
\end{tabular}

Se puntúa de 0 a 3 en cada uno de los apartados (mínimo 0, máximo 9).

\begin{tabular}{|l|c|c|c|}
\hline Gravedad & PS & PEF* $^{*}$ & Sat O $_{2}$ \\
\hline Leve & $0-3$ & $>80 \%$ & $>94$ \\
\hline Moderada & $4-6$ & $60-80 \%$ & $91-94$ \\
\hline Grave & $7-9$ & $<60 \%$ & $<91$ \\
\hline
\end{tabular}

*Tras la administración de una dosis de broncodilatador.

En caso de discordancia entre PS, PEF y Sat $\mathrm{O}_{2}$, se clasificará con el de mayor gravedad. 
- Valoración de la gravedad y/o grado de control del asma, en el momento del diagnóstico y al menos una vez al año.

- Estudio de la función respiratoria, en el momento del diagnóstico y al menos una vez al año.

- Estudio de la sensibilización alérgica, en el momento del diagnóstico o en alguna de las revisiones posteriores.

- Consejo sobre medidas de control ambiental, por lo menos una vez al año.

- Revisión del tratamiento farmacológico, al inicio del tratamiento y por lo menos una vez al año.

- Revisión de la técnica de inhalación, al inicio del tratamiento y por lo menos una vez al año.

\section{BIBLIOGRAFÍA}

1. Global Strategy for Asthma Management and Prevention. Global Initiative for Asthma (GINA) 2012. Available from www.ginasthma.org/.Global strategy for asthma management and prevention for adults and children older than 5 years. Global Initiative for Asthma [en línea] [actualizado en 2012; consultado el 24/04/ 2013]. Disponible en www.ginasthma.org

2. Cobos N. Asma: del síntoma al remodelamiento. An Pediatr. 2003;58(Supl 1):89-96.

3. British Thotacic Society. Scottish Intercollegiate Guidelines Network. British Guideline on the management of asthma [en línea] [actualizado en 2012; consultado el 24/04/2013]. Disponible en www.sign. ac.uk/guidelines/fulltext/101/index.html

4. Expert Panel Report 3 (EPR3). Guidelines for the diagnosis and management of asthma 2007 [en línea] [consultado el 24/04/2013]. Disponible en www.nhlbi.nih.gov/guidelines/asthma/asthgdln.htm

5. Guía española para el manejo del asma (GEMA) 2009 [en línea] [consultado el 24/04/2013]. Disponible en www.gemasma.com
- Educación terapéutica y plan de acción por escrito sobre actuación ante una crisis, empeoramiento de la enfermedad y los criterios para solicitar ayuda médica, en el momento del diagnóstico y al menos una vez al año.

\section{CONFLICTO DE INTERESES}

La autora declara no presentar conflictos de intereses en relación con la preparación y publicación de este artículo.

\section{ABREVIATURAS}

AP: Atención Primaria • BAL: Agonista $\beta_{2}$ adrenérgico de acción larga • GCl: glucocorticoides inhalados • GEMA: Guía Española de Manejo del Asma • GINA: Iniciativa Global para el Asma • GPC: guías de práctica clínica • IPA: Índice Predictivo de Asma.
6. Castro-Rodríguez JA, Rodrigo GJ. Efficacy of inhaled corticosteroids in infants and preschoolers with recurrent wheezing and asthma: a systematic review with meta-analysis. Pediatrics. 2009;123(3): e519-25.

7. Cano Garcinuño A, Carvajal Urueña I, Díaz Vázquez CA, Mora Gandarillas I, Mola Caballero de Rodas P, García Merino A, et al. Control del asma en niños: validez del cuestionario CAN y su relación con la función pulmonar y el óxido nítrico exhalado. Bol Pediatr. 2011;51:39-46.

8. Castillo Laita JA, de Benito Fernández J, Escribano Montaner A, Fernández Benítez M, García de la Rubia S, Garde Garde J, et al. Consenso sobre el tratamiento de asma en pediatría. An Pediatr (Barc). 2007;67:253-7.

9. Pedersen SE, Hurd SS, Lemanske RF, Becker A, Zar HJ, Sly PD, et al. Global Strategy for the diagnosis and management of asthma in children 5 years and younger. Pediatr Pulmonol. 2011;46:1-17.

10. Smith SR, Baty JD, Hodge D. Validation on the pulmonary score: an asthma severity score for children. Acad Emerg Med. 2002;9(2):99-104. 